\title{
Application of the Multiple Exp-Function, Cross-Kink, Periodic- Kink, Solitary Wave Methods, and Stability Analysis for the CDG Equation
}

\author{
Haifa Bin Jebreen $\mathbb{D i D}^{1}$ and Yurilev Chalco-Cano $\mathbb{D D}^{2}$ \\ ${ }^{1}$ Mathematics Department, College of Science, King Saud University, Riyadh, Saudi Arabia \\ ${ }^{2}$ Departamento de Mathematica, Universidad de Tarapaca, Casilla $7 D$, Arica, Chile \\ Correspondence should be addressed to Haifa Bin Jebreen; hjebreen@ksu.edu.sa
}

Received 23 November 2020; Revised 16 December 2020; Accepted 31 December 2020; Published 16 January 2021

Academic Editor: Sachin Kumar

Copyright (@) 2021 Haifa Bin Jebreen and Yurilev Chalco-Cano. This is an open access article distributed under the Creative Commons Attribution License, which permits unrestricted use, distribution, and reproduction in any medium, provided the original work is properly cited.

\begin{abstract}
In this article, the exact wave structures are discussed to the Caudrey-Dodd-Gibbon equation with the assistance of Maple based on the Hirota bilinear form. It is investigated that the equation exhibits the trigonometric, hyperbolic, and exponential function solutions. We first construct a combination of the general exponential function, periodic function, and hyperbolic function in order to derive the general periodic-kink solution for this equation. Then, the more periodic wave solutions are presented with more arbitrary autocephalous parameters, in which the periodic-kink solution localized in all directions in space. Furthermore, the modulation instability is employed to discuss the stability of the available solutions, and the special theorem is also introduced. Moreover, the constraint conditions are also reported which validate the existence of solutions. Furthermore, 2dimensional graphs are presented for the physical movement of the earned solutions under the appropriate selection of the parameters for stability analysis. The concluded results are helpful for the understanding of the investigation of nonlinear waves and are also vital for numerical and experimental verification in engineering sciences and nonlinear physics.
\end{abstract}

\section{Introduction}

It is known that these exact solutions of nonlinear evolution equations (NLEEs), especially the soliton solutions [1-3], can be given by using a variety of different methods $[4,5]$, such as Jacobi elliptic function expansion method [6], inverse scattering transformation (IST) [7, 8], Darboux transformation (DT) [9], extended generalized DT [10], Lax pair (LP) [11], Lie symmetry analysis [12], Hirota bilinear method [13], and others $[14,15]$. The Hirota bilinear method is an efficient tool to construct exact solutions of NLEEs, and there exists plenty of completely integrable equations which are studied in this way. For instance, generalized bilinear equations [16], the lump-type solutions in a homogenous-dispersive medium [17], the $(2+1)$-dimensional $\mathrm{KdV}$ equation [18], the $(3+1)$-D potential-YTSF equation [19], the generalized
BKP equation [20], the $(3+1)$-dimensional BKPBoussinesq equation [21], the $(3+1)$ dimensional generalized KP-Boussinesq equation [22], the $(2+1)$-dimensional integrable Boussinesq model [23], and the $(2+1)$-dimensional Breaking Soliton equation [24, 25]. More recently, lump waves and rogue waves have attracted a growing amount of attention, and many theoretical and experimental studies of lump waves are mentioned [26-33]. A novel method for finding the special rogue waves with predictability of NLEEs is proposed by using the Hirota bilinear method by powerful researchers in Refs. [34, 35], in which some results are very helpful for us to study some physical phenomena in engineering.

The Caudrey-Dodd-Gibbon equation introduced by Aiyer et al. [36] who describes the inelastic interactions between the solitary waves under strong physical contexts 
in certain integrable or nonintegrable systems and has been investigated the related dynamic behavior [37], which reads

$$
\Phi_{t}+\Phi_{x x x x x}+30 \Phi \Phi_{x x x}+30 \Phi_{x} \Phi_{x x}+180 \Phi^{2} \Phi_{x}=0
$$

In 2006, the tanh solutions of the equation [38] and, in 2008, the multiple-soliton solutions utilizing the Hirota bilinear method combined with the simplified Hereman method [39] for the above equation are derived by Wazwaz. Also, the physical comprehension of Equation (1) was demonstrated by plenty of scholars who investigated its solitary type solutions and given in Refs. [40] and [41]. The homotopy perturbation method has been utilized to find solutions for the aforementioned equation [42-44]. Based on the obtained transformation of integrating Equation (1), we get to the following nonlinear PDE [45]:

$$
\Lambda_{t}+60 \Lambda_{x}^{3}+30 \Lambda_{x} \Lambda_{x x x}+\Lambda_{x x x x x}=0
$$

According to [46], the Hirota bilinear from of the CDG equation reads

$$
\Lambda=2(\ln \Gamma)_{x},\left(\Phi=\Lambda_{x}\right)
$$

and, also by applying the dependent variable transformation, turns into the Hirota bilinear form

$$
\left(D_{x t}+D_{x}^{6}\right) \Gamma \cdot \Gamma=0
$$

where $D$ is a bilinear operator. By deeming the $D$-operator defined with the aid of the functions $\Gamma_{1}$ and $\Gamma_{2}$, we get to the following relation:

$$
D_{x}^{\pi_{1}} D_{t}^{\pi_{2}}\left(\Gamma_{1} \cdot \Gamma_{2}\right)=\left.\left(\frac{\partial}{\partial x}-\frac{\partial}{\partial x^{\prime}}\right)^{\pi_{1}}\left(\frac{\partial}{\partial t}-\frac{\partial}{\partial t^{\prime}}\right)^{\pi_{2}} \Gamma_{1}(x, t) \cdot \Gamma_{2}\left(x^{\prime}, t^{\prime}\right)\right|_{x} ^{\prime=x, t^{\prime}=t} .
$$

With the help of the transformation Equation (3), the general periodic-kink solutions of Equation (1) can be given. We get to the bilinear form the of $\Gamma$ as

$$
\Gamma \Gamma_{x t}-\Gamma_{x} \Gamma_{t}+\Gamma \Gamma_{x x x x x x}-6 \Gamma_{x} \Gamma_{x x x x x}+15 \Gamma_{x x} \Gamma_{x x x x}-10 \Gamma_{x x x}^{2}=0 .
$$

Moreover, the stability analysis and the more general periodic-wave solutions and special rogue waves with predictability are investigated in our paper, which have never been studied. Various types of studies were investigated by capable authors in which some of them can be mentioned, for example, the Caudrey-Dodd-Gibbon equation [47], the pZK equation using Lie point symmetries [48], groupinvariant solutions of the $(3+1)$-dimensional generalized KP equation [49], optimal system and dynamics of solitons for a higher-dimensional Fokas equation [50], dynamics of solitons for $(2+1)$-dimensional NNV equations [51], the combined MCBS-nMCBS equation [52], Lie symmetry reductions for $(2+1)$-dimensional Pavlov equation [53], Schrödinger-Hirota equation with variable coefficients [54], the $(2+1)$-dimension paraxial wave equation [55], the fractional Drinfeld-Sokolov-Wilson equation [56], the $(3+1)$-dimensional extended Jimbo-Miwa equations [57], and a high-order partial differential equation with fractional derivatives [58]. In the valuable work, the capable authors studied the periodic wave solutions and stability analysis for the KP-BBM equation [59] and breather and periodic wave solution for generalized Bogoyavlensky-Konopelchenko equation [60] with the aid of Hirota operator.

To make this paper more self-contained, a combination of general exponential function, periodic function, and hyperbolic function of the $(3+1)$-dimensional CDG equation is constructed with the help of a bilinear operator, which is crucial to obtain the periodic-wave solution of Equation (1). Based on the Hirota bilinear form Equation (6), the general periodic-wave solution is derived in Section 2 and the novel periodic solutions which can be arisen with twenty one classes. In Section 4, we shall investigate the stability analysis to obtain the modulation stability spectrum of this equation. The final section will be reserved for the conclusions and the discussions.

\section{Multiple Exp-Function Method}

In this section, according to [61-63] so that it can be further employed to the nonlinear partial differential equation (NLPDE) in order to furnish its exact solutions, it can be presented as:

Step 1. The following is the NLPDE:

$$
\mathscr{P}\left(x, y, t, \Psi, \Psi_{x}, \Psi_{y}, \Psi_{z}, \Psi_{t}, \Psi_{x x}, \Psi_{t t}, \cdots\right)=0 .
$$

We commence a sequence of new variables $\xi_{i}=\xi_{i}(x, t)$, $1 \leq i \leq n$, by solvable PDEs, for example, the linear ones,

$$
\xi_{i, x}=\alpha_{i} \xi_{i}, \xi_{i, t}=\delta_{i} \xi_{i}, \quad 1 \leq i \leq n,
$$

where $\alpha_{i}, 1 \leq i \leq n$, is the angular wave number and $\delta_{i}, 1 \leq i$ $\leq n$, is the wave frequency. It should be pointed that this is frequently the initiating step for constructing the exact solutions to NLPDEs, and moreover, solving such linear equations redounds to the exponential function solutions:

$$
\xi_{i}=\omega_{i} e^{\theta_{i}}, \theta_{i}=\alpha_{i} x-\delta_{i} t, \quad 1 \leq i \leq n,
$$

in which $\omega_{i}, 1 \leq i \leq n$, are undetermined constants.

Step 2. Determine the solution of Equation (7) as the following form in terms of the new variables $\xi_{i}, 1 \leq i \leq n$ :

$$
\Psi(x, t)=\frac{\Delta\left(\xi_{1}, \xi_{2}, \cdots, \xi_{n}\right)}{\Omega\left(\xi_{1}, \xi_{2}, \cdots, \xi_{n}\right)}, \quad \Delta=\sum_{r, s=1}^{n} \sum_{i, j=0}^{M} \Delta_{r s, i j} \xi_{r}^{i} \xi_{s}^{j}, \Omega=\sum_{r, s=1}^{n} \sum_{i, j=0}^{N} \Omega_{r s, i j} \xi_{r}^{i} \xi_{s}^{j},
$$

in which $\Delta_{r s, i j}$ and $\Omega_{r s, i j}$ are the amounts to be settled. Appending Equation (10) into Equation (7) and ordering the numerator of the rational function to zero, we can achieve 
a series of nonlinear algebraic equations about the variables $\alpha_{i}, \delta_{i}, \Delta_{r s, i j}$ and $\Omega_{r s, i j}$. Solving the solutions for these nonlinear algebraic equations and putting these solutions into Equation (10), the multiple soliton solutions to Equation (7) can be obtained in the below form as

$$
\Psi(x, t)=\frac{\Delta\left(\omega_{1} e^{\alpha_{1} x-\delta_{1} t}, \cdots, \omega_{n} e^{\alpha_{n} x-\delta_{n} t}\right)}{\Omega\left(\omega_{1} e^{\alpha_{1} x-\delta_{1} t}, \cdots, \omega_{n} e^{\alpha_{n} x-\delta_{n} t}\right)},
$$

in which $\Omega \neq 0$, and also, we have

$$
\begin{aligned}
& \Delta_{t}=\sum_{i=1}^{n} \Delta_{\xi_{i}} \xi_{i, t}, \Omega_{t}=\sum_{i=1}^{n} \Omega_{\xi_{i}} \xi_{i, t}, \Delta_{x}=\sum_{i=1}^{n} \Delta_{\xi_{i}} \xi_{i, x}, \Omega_{x}=\sum_{i=1}^{n} \Omega_{\xi_{i}} \xi_{i, x}, \\
& \Psi_{t}=\frac{\Omega \sum_{i=1}^{n} \Delta_{\xi_{i}} \xi_{i, t}-\Delta \sum_{i=1}^{n} \Omega_{\xi_{i}} \xi_{i, t}}{\Omega^{2}}, \Psi_{x}=\frac{\Omega \sum_{i=1}^{n} \Delta_{\xi_{i}} \xi_{i, x}-\Delta \sum_{i=1}^{n} \Omega_{\xi_{i}} \xi_{i, x}}{\Omega^{2}} .
\end{aligned}
$$

\section{Multiple Soliton Solutions for the CDG Equation}

3.1. Set I: One-Wave Solution. We start up with one-wave function based on the explanation in Step 2 in the previous section, we deem that Equation (1) has the below form of one-wave solution as

$$
\Psi(x, t)=\frac{\eta_{1}}{\eta_{2}}, \quad \eta_{1}=\sigma_{1}+\sigma_{2} e^{\alpha_{1} x-\delta_{1} t}, \eta_{2}=1+\rho_{1}+\rho_{2} e^{\alpha_{1} x-\delta_{1} t},
$$

in which $\rho_{1}, \rho_{2}, \sigma_{1}$, and $\sigma_{2}$ are the unfound constants. Plugging (13) into Equation (1), we get to the following cases:

Case 1.

$$
\begin{aligned}
& \alpha_{1}=\alpha_{1}, \beta_{1}=\beta_{1}, \rho_{1}=\frac{\rho_{2} \sigma_{1}-\sigma_{2}}{\sigma_{2}}, \rho_{2}=\rho_{2}, \\
& \sigma_{1}=\sigma_{1}, \sigma_{2}=\sigma_{2}, \delta_{1}=\alpha_{1}^{5} .
\end{aligned}
$$

Case 2.

$$
\begin{aligned}
& \alpha_{1}=\alpha_{1}, \beta_{1}=\beta_{1}, \rho_{1}=-1, \rho_{2}=\rho_{2} \\
& \sigma_{1}=\sigma_{1}, \sigma_{2}=\sigma_{2}, \delta_{1}=\alpha_{1}^{5}
\end{aligned}
$$

Case 3.

$$
\begin{aligned}
& \alpha_{1}=\alpha_{1}, \beta_{1}=\beta_{1}, \rho_{1}=\rho_{1}, \rho_{2}=\frac{\sigma_{2}\left(1+\rho_{1}\right)}{\sigma_{1}}, \\
& \sigma_{1}=\sigma_{1}, \sigma_{2}=\sigma_{2}, \delta_{1}=\delta_{1} .
\end{aligned}
$$

For example, the resulting one-wave solution for Cases 1 to 3 will be read, respectively, as

$$
\begin{aligned}
& \Psi_{1}(x, t)=\frac{\sigma_{1}+\sigma_{2} e^{-t \alpha_{1}{ }^{5}+x \alpha_{1}}}{1+\left(\left(\rho_{2} \sigma_{1}-\sigma_{2}\right) / \sigma_{2}\right)+\rho_{2} e^{-t \alpha_{1}{ }^{5}+x \alpha_{1}}}, \\
& \Psi_{2}(x, t)=\frac{\sigma_{1}+\sigma_{2} e^{-t \alpha_{1}{ }^{5}+x \alpha_{1}}}{\rho_{2} e^{-t \alpha_{1}{ }^{5}+x \alpha_{1}}}, \\
& \Psi_{1}(x, t)=\frac{\sigma_{1}+\sigma_{2} e^{-t \delta_{1}+x \alpha_{1}}}{1+\rho_{1}+\left(\left(\sigma_{2}\left(1+\rho_{1}\right) e^{-t \delta_{1}+x \alpha_{1}}\right) / \sigma_{1}\right)} .
\end{aligned}
$$

3.2. Set II: Two-Wave Solutions. We start up with two-wave functions based on the explanations in Step 2 in the previous section; we deem that Equation (1) has the bellow form of two-wave solutions as

$$
\begin{gathered}
\Psi(x, t)=\frac{\eta_{1}}{\eta_{2}} \\
\eta_{1}=\rho_{0}+\rho_{1} e^{\alpha_{1} x-\delta_{1} t}+\rho_{2} e^{\alpha_{2} x-\delta_{2} t}+\rho_{1} \rho_{2} \rho_{12} e^{\left(\alpha_{1}+\alpha_{2}\right) x-\left(\delta_{1}+\delta_{2}\right) t} \\
\eta_{2}=1+\sigma_{1} e^{\alpha_{1} x-\delta_{1} t}+\sigma_{2} e^{\alpha_{2} x-\delta_{2} t}+\sigma_{1} \sigma_{2} \sigma_{12} e^{\left(\alpha_{1}+\alpha_{2}\right) x-\left(\delta_{1}+\delta_{2}\right) t}
\end{gathered}
$$

Plugging (18) along with (19) into Equation (1), we gain the following cases:

Case 1.

$$
\begin{aligned}
& \alpha_{1}=0, \alpha_{2}=\alpha_{2}, \delta_{1}=\delta_{1}, \delta_{1}=\delta_{1}, \rho_{0}=\frac{\rho_{2}}{\sigma_{2}}, \\
& \rho_{1}=0, \rho_{2}=\rho_{2}, \rho_{12}=\rho_{12}, \sigma_{1}=\sigma_{1}, \sigma_{2}=\sigma_{2}, \sigma_{12}=1 .
\end{aligned}
$$

Case 2.

$$
\begin{aligned}
& \alpha_{1}=0, \alpha_{2}=\alpha_{2}, \delta_{1}=\delta_{1}, \delta_{2}=\delta_{2}, \rho_{0}=\frac{1}{\rho_{12}} \\
& \rho_{1}=\rho_{1}, \rho_{2}=\frac{\sigma_{2}}{\rho_{12}}, \rho_{12}=\rho_{12}, \sigma_{1}=0, \sigma_{2}=\sigma_{2}, \sigma_{12}=\sigma_{12} .
\end{aligned}
$$

Case 3.

$$
\begin{aligned}
& \alpha_{1}=0, \alpha_{2}=\alpha_{2}, \delta_{1}=\delta_{1}, \delta_{2}=\alpha_{2}^{5}, \rho_{0}=\frac{1}{\rho_{12}} \\
& \rho_{1}=\rho_{1}, \rho_{2}=\rho_{2}, \rho_{12}=\rho_{12}, \sigma_{1}=\sigma_{1}, \sigma_{2}=0, \sigma_{12}=\sigma_{12} .
\end{aligned}
$$

Case 4.

$$
\begin{aligned}
& \alpha_{1}=0, \alpha_{2}=\alpha_{2}, \delta_{1}=\delta_{1}, \delta_{2}=\delta_{2}, \rho_{0}=\frac{\rho_{2}}{\sigma_{2}} \\
& \rho_{1}=\rho_{1}, \rho_{2}=\rho_{2}, \rho_{12}=\frac{\sigma_{2}}{\rho_{2}}, \sigma_{1}=\sigma_{1}, \sigma_{2}=\sigma_{2}, \sigma_{12}=1 .
\end{aligned}
$$


Case 5.

$$
\begin{gathered}
\alpha_{1}=\alpha_{1}, \alpha_{2}=\alpha_{2}, \delta_{1}=\alpha_{1}^{5}, \delta_{2}=\alpha_{2}^{5}, \\
\rho_{0}=\frac{\alpha_{1}{ }^{4}-3 \alpha_{1}^{3} \alpha_{2}+4 \alpha_{1}{ }^{2} \alpha_{2}{ }^{2}-3 \alpha_{1} \alpha_{2}{ }^{3}+\alpha_{2}{ }^{4}}{\rho_{12}\left(\alpha_{1}{ }^{2}+\alpha_{1} \alpha_{2}+\alpha_{2}{ }^{2}\right)\left(\alpha_{1}+\alpha_{2}\right)^{2}}, \\
\rho_{1}=\rho_{1}, \rho_{2}=\rho_{2}, \rho_{12}=\rho_{12}, \sigma_{1}=0, \sigma_{2}=0, \sigma_{12}=\sigma 12 .
\end{gathered}
$$

Case 6.

$$
\begin{gathered}
\alpha_{1}=\alpha_{1}, \alpha_{2}=\alpha_{2}, \delta_{1}=\alpha_{1}^{5}, \delta_{2}=\delta_{2}, \rho_{0}=\frac{1}{\rho_{12}}, \\
\rho_{1}=\rho_{1}, \rho_{2}=\frac{\sigma_{2}}{\rho_{12}}, \rho_{12}=\rho_{12}, \\
\sigma_{1}=0, \sigma_{2}=\sigma_{2}, \sigma_{12}=\sigma 12 .
\end{gathered}
$$

Case 7.

$$
\begin{aligned}
& \alpha_{1}=\alpha_{1}, \alpha_{2}=\alpha_{2}, \delta_{1}=\delta_{1}, \delta_{2}=\delta_{2}, \rho_{0}=\frac{\rho_{2}}{\sigma_{2}} \\
& \rho_{1}=\frac{\rho_{2} \sigma_{1}}{\sigma_{2}}, \rho_{2}=\rho_{2}, \rho_{12}=\frac{\sigma_{2} \sigma_{12}}{\rho_{2}}, \sigma_{1}=0, \sigma_{2}=\sigma_{2}, \sigma_{12}=\sigma_{12} .
\end{aligned}
$$

For example, the resulting two-wave solution for Cases 1-7 will be read, respectively, as

$$
\begin{aligned}
\Psi_{1}(x, t)= & \frac{\left(\rho_{2} / \sigma_{2}\right)+\rho_{2} e^{-t \delta_{2}+x \alpha_{2}}}{1+\sigma_{1} e^{-t \delta_{1}}+\sigma_{2} e^{-t \delta_{2}+x \alpha_{2}}+\sigma_{1} \sigma_{2} e^{-t \delta_{1}-t \delta_{2}+x \alpha_{2}}}, \\
\Psi_{2}(x, t)= & \frac{\rho_{12}{ }^{-1}+\rho_{1} e^{-t \delta_{1}}+\left(\left(\sigma_{2} e^{-t \delta_{2}+x \alpha_{2}}\right) / \rho_{12}\right)+\rho_{1} \sigma_{2} e^{-t \delta_{1}-t \delta_{2}+x \alpha_{2}}}{1+\sigma_{2} e^{-t \delta_{2}+x \alpha_{2}}}, \\
\Psi_{3}(x, t)= & \frac{\rho_{12}{ }^{-1}+\rho_{1} e^{-t \delta_{1}}+\rho_{2} e^{-t \alpha_{2}{ }^{5}+x \alpha_{2}}+\rho_{1} \rho_{2} \rho_{12} e^{-t \alpha_{2}{ }^{5}-t \delta_{1}+x \alpha_{2}}}{1+\sigma_{1} e^{-t \delta_{1}}}, \\
\Psi_{4}(x, t)= & \frac{\left(\rho_{2} / \sigma_{2}\right)+\rho_{1} e^{-t \delta_{1}}+\rho_{2} e^{-t \delta_{2}+x \alpha_{2}}+\rho_{1} \sigma_{2} e^{-t \delta_{1}-t \delta_{2}+x \alpha_{2}}}{1+\sigma_{1} e^{-t \delta_{1}}+\sigma_{2} e^{-t \delta_{2}+x \alpha_{2}}+\sigma_{1} \sigma_{2} e^{-t \delta_{1}-t \delta_{2}+x \alpha_{2}}}, \\
\Psi_{5}(x, t)= & \frac{\alpha_{1}{ }^{4}-3 \alpha_{1}{ }^{3} \alpha_{2}+4 \alpha_{1}{ }^{2} \alpha_{2}{ }^{2}-3 \alpha_{1} \alpha_{2}{ }^{3}+\alpha_{2}{ }^{4}}{\rho_{12}\left(\alpha_{1}{ }^{2}+\alpha_{1} \alpha_{2}+\alpha_{2}{ }^{2}\right)\left(\alpha_{1}+\alpha_{2}\right)^{2}} \\
& +\rho_{1} e^{-t \alpha_{1}{ }^{5}+x \alpha_{1}}+\rho_{2} e^{-t \alpha_{2}{ }^{5}+x \alpha_{2}}+\rho_{1} \rho_{2} \rho_{12} e^{-t \alpha_{1}{ }^{5}-t \alpha_{2}{ }^{5}+x \alpha_{1}+x \alpha_{2}}, \\
\Psi_{6}(x, t)= & \frac{\rho_{12}{ }^{-1}+\rho_{1} e^{-t \alpha_{1}{ }^{5}+x \alpha_{1}}+\left(\left(\sigma_{2} e^{-t \delta_{2}+x \alpha_{2}}\right) / \rho_{12}\right)+\rho_{1} \sigma_{2} e^{-t \alpha_{1}{ }^{5}-t \delta_{2}+x \alpha_{1}+x \alpha_{2}}}{1+\sigma_{2} e^{-t \delta_{2}+x \alpha_{2}}}, \\
\Psi_{7}(x, t)= & \frac{\left(\rho_{2} / \sigma_{2}\right)+\left(\left(\rho_{2} \sigma_{1} e^{-t \delta_{1}+x \alpha_{1}}\right) / \sigma_{2}\right)+\rho_{2} e^{-t \delta_{2}+x \alpha_{2}}+\rho_{2} \sigma_{1} \sigma_{12} e^{-t \delta_{1}-t \delta_{2}+x \alpha_{1}+x \alpha_{2}}}{1+\sigma_{1} e^{-t \delta_{1}+x \alpha_{1}}+\sigma_{2} e^{-t \delta_{2}+x \alpha_{2}}+\sigma_{1} \sigma_{2} \sigma_{12} e^{-t \delta_{1}-t \delta_{2}+x \alpha_{1}+x \alpha_{2}}} .
\end{aligned}
$$

3.3. Set III: Three-Wave Solutions. We start up with threewave functions according to the given explanations in Step 2 in the previous section, we deem that Equation (1) has the below form of three-wave solutions as

$$
\Psi(x, t)=\frac{d f\left(\eta_{1}\right) / d x}{\eta_{1}}
$$

$$
\begin{aligned}
\eta_{1}= & +\rho_{1} e^{\Lambda_{1}}+\rho_{2} e^{\Lambda_{2}}+\rho_{3} e^{\Lambda_{3}} \\
& +\rho_{1} \rho_{2} \rho_{12} e^{\Lambda_{1}+\Lambda_{2}} \\
& +\rho_{1} \rho_{3} \rho_{13} e^{\Lambda_{1}+\Lambda_{3}}+\rho_{2} \rho_{3} \rho_{23} e^{\Lambda_{2}+\Lambda_{3}} \\
& +\rho_{1} \rho_{2} \rho_{3} \rho_{12} \rho_{13} \rho_{23} e^{\Lambda_{1}+\Lambda_{2}+\Lambda_{3}},
\end{aligned}
$$

in which $\Lambda_{i}=\alpha_{i} x-\delta_{i} t, i=1,2,3$. Appending (29) along with (30) into Equation (1), we obtain the following case:

$$
\begin{aligned}
& \alpha_{i}=\alpha_{i}, \delta_{i}=\alpha_{i}^{5}, \quad i=1,2,3, \\
& \rho_{i j}=\rho_{i j}, \quad i, j=1,2,3, i \neq j .
\end{aligned}
$$

Therefore, three-wave solution will be as

$$
\begin{aligned}
& \Psi_{1}(x, t)=\rho_{1} \alpha_{1} e^{-t \alpha_{1}{ }^{5}+x \alpha_{1}}+\rho_{2} \alpha_{2} e^{-t \alpha_{2}{ }^{5}+x \alpha_{2}}+\rho_{3} \alpha_{3} e^{-t \alpha_{3}{ }^{5}+x \alpha_{3}} \\
& \quad+\rho_{1} \rho_{2} \rho_{12}\left(\alpha_{1}+\alpha_{2}\right) e^{-t \alpha_{1}{ }^{5}-t \alpha_{2}{ }^{5}+x \alpha_{1}+x \alpha_{2}} \\
& \quad+\rho_{1} \rho_{3} \rho_{13}\left(\alpha_{1}+\alpha_{3}\right) e^{-t \alpha_{1}{ }^{5}-t \alpha_{3}{ }^{5}+x \alpha_{1}+x \alpha_{3}} \\
& \quad+\rho_{2} \rho_{3} \rho_{23}\left(\alpha_{2}+\alpha_{3}\right) e^{-t \alpha_{2}{ }^{5}-t \alpha_{3}{ }^{5}+x \alpha_{2}+x \alpha_{3}} \\
& \quad+\rho_{1} \rho_{2} \rho_{3} \rho_{12} \rho_{13} \rho_{23}\left(\alpha_{1}+\alpha_{2}+\alpha_{3}\right) e^{-t \alpha_{1}{ }^{5}-t \alpha_{2}{ }^{5}-t \alpha_{3}{ }^{5}+x \alpha_{1}+x \alpha_{2}+x \alpha_{3}} / \\
& +\left(1+\rho_{1} e^{-t \alpha_{1}{ }^{5}+x \alpha_{1}}+\rho_{2} e^{-t \alpha_{2}{ }^{5}+x \alpha_{2}}+\rho_{3} e^{-t \alpha_{3}{ }^{5}+x \alpha_{3}}\right. \\
& +\rho_{1} \rho_{2} \rho_{12} e^{-t \alpha_{1}{ }^{5}-t \alpha_{2}{ }^{5}+x \alpha_{1}+x \alpha_{2}}+\rho_{1} \rho_{3} \rho_{13} e^{-t \alpha_{1}{ }^{5}-t \alpha_{3}{ }^{5}+x \alpha_{1}+x \alpha_{3}} \\
& +\rho_{2} \rho_{3} \rho_{23} e^{-t \alpha_{2}{ }^{5}-t \alpha_{3}{ }^{5}+x \alpha_{2}+x \alpha_{3}} \\
& \left.+\rho_{1} \rho_{2} \rho_{3} \rho_{12} \rho_{13} \rho_{23} e^{-t \alpha_{1}{ }^{5}-t \alpha_{2}{ }^{5}-t \alpha_{3}{ }^{5}+x \alpha_{1}+x \alpha_{2}+x \alpha_{3}}\right) .
\end{aligned}
$$

3.4. Cross-Kink Solutions. Here, we will consider the cross-kink wave solution with selecting the below function which for Equation (1) has been taken as

$$
\begin{gathered}
f=\exp \left(\tau_{1}\right)+\theta_{10} \exp \left(-\tau_{1}\right)+\sinh \left(\tau_{2}\right) \\
+\sin \left(\tau_{3}\right)+\theta_{11}, \tau_{1}=\theta_{1} x+\theta_{2} t \\
+\theta_{3}, \tau_{2}=\theta_{4} x+\theta_{5} t \\
+\theta_{6}, \tau_{3}=\theta_{7} x+\theta_{8} t+\theta_{9}, \\
\Psi(x, t)=\ln (f)_{x x},
\end{gathered}
$$

where $\theta_{i}, i=1, \cdots, 11$, are undetermined amounts which should be detected. Appending (34) into Equation (1) and afterwards collecting the coefficients, we obtain the following consequences:

Case 1

$$
\begin{aligned}
& \theta_{1}=\theta_{4}=i \theta_{7}, \theta_{2}=\theta_{5}=-16 i \theta_{7}^{5}, \\
& \theta_{8}=-16 \theta_{7}^{5}, \theta_{11}=0, i^{2}=-1 .
\end{aligned}
$$


Substituting (35) into (33) and (34), we achieve a crosskink wave solution of Equation (1) as follows:

$$
\begin{aligned}
f= & e^{-16 i \theta_{7}^{5} t+i \theta_{7} x+\theta_{3}}+\theta_{10} e^{16 i \theta_{7}^{5} t-i \theta_{7} x-\theta_{3}}-\sinh \left(16 i \theta_{7}^{5} t-i \theta_{7} x-\theta_{6}\right)-\sin \left(16 \theta_{7}^{5} t-\theta_{7} x-\theta_{9}\right), \\
\Psi_{1}= & \frac{-\theta_{7}^{2} e^{-16 i \theta_{7}^{5} t+i \theta_{7} x+\theta_{3}}-\theta_{10} \theta_{7}{ }^{2} e^{16 i \theta_{7}^{5} t-i \theta_{7} x-\theta_{3}}+\sinh \left(16 i \theta_{7}^{5} t-i \theta_{7} x-\theta_{6}\right) \theta_{7}^{2}+\sin \left(16 \theta_{7}^{5} t-\theta_{7} x-\theta_{9}\right) \theta_{7}^{2}}{e^{-16 i \theta_{7}^{5} t+i \theta_{7} x+\theta_{3}}+\theta_{10} e^{16 i \theta_{7}^{5} t-i \theta_{7} x-\theta_{3}}-\sinh \left(16 i \theta_{7}^{5} t-i \theta_{7} x-\theta_{6}\right)-\sin \left(16 \theta_{7}^{5} t-\theta_{7} x-\theta_{9}\right)} \\
& -\frac{\left(i \theta_{7} e^{-16 i \theta_{7}{ }^{5} t+i \theta_{7} x+\theta_{3}}-i \theta_{10} \theta_{7} e^{16 i \theta_{7}^{5} t-i \theta_{7} x-\theta_{3}}+i \cosh \left(16 i \theta_{7}^{5} t-i \theta_{7} x-\theta_{6}\right) \theta_{7}+\cos \left(16 \theta_{7}^{5} t-\theta_{7} x-\theta_{9}\right) \theta_{7}\right)^{2}}{\left(e^{-16 i \theta_{7}{ }^{5} t+i \theta_{7} \mathrm{x}+\theta_{3}}+\theta_{10} e^{16 i \theta_{7}{ }^{5} t-i \theta_{7} x-\theta_{3}}-\sinh \left(16 i \theta_{7}^{5} t-i \theta_{7} x-\theta_{6}\right)-\sin \left(16 \theta_{7}^{5} t-\theta_{7} x-\theta_{9}\right)\right)^{2}} .
\end{aligned}
$$

Case 2.

$$
\begin{aligned}
& \theta_{1}=\sqrt{3} \theta_{7}=i \sqrt{3} \theta_{4}, \theta_{2}=-\sqrt{3} \theta_{8}=16 \sqrt{3} i \theta_{4}^{5}, \\
& \theta_{5}=-16 \theta_{4}^{5}, \theta_{10}=0, \theta_{11}=0, i^{2}=-1 .
\end{aligned}
$$

$$
\begin{aligned}
f= & e^{16 \sqrt{3} \theta_{4}{ }^{5} i t+\sqrt{3} i \theta_{4} x+\theta_{3}}-\sinh \left(16 t \theta_{4}{ }^{5}-x \theta_{4}-\theta_{6}\right)-\sin \left(16 \theta_{4}{ }^{5} i t-i \theta_{4} x-\theta_{9}\right), \\
\Psi_{1}= & \frac{3 i^{2} \theta_{4}{ }^{2} e^{16 \sqrt{3} \theta_{4}{ }^{5} i t+\sqrt{3} i \theta_{4} x+\theta_{3}}-\sinh \left(16 t \theta_{4}{ }^{5}-x \theta_{4}-\theta_{6}\right) \theta_{4}{ }^{2}+\sin \left(16 \theta_{4}{ }^{5} i t-i \theta_{4} x-\theta_{9}\right) i^{2} \theta_{4}{ }^{2}}{e^{16 \sqrt{3} \theta_{4}{ }^{5} i t+\sqrt{3} i \theta_{4} x+\theta_{3}}-\sinh \left(16 t \theta_{4}{ }^{5}-x \theta_{4}-\theta_{6}\right)-\sin \left(16 \theta_{4}{ }^{5} i t-i \theta_{4} x-\theta_{9}\right)} \\
& -\frac{\left(\sqrt{3} i \theta_{4} e^{16 \sqrt{3} \theta_{4}{ }^{5} i t+\sqrt{3} i \theta_{4} x+\theta_{3}}+\cosh \left(16 t \theta_{4}{ }^{5}-x \theta_{4}-\theta_{6}\right) \theta_{4}+\cos \left(16 \theta_{4}{ }^{5} i t-i \theta_{4} x-\theta_{9}\right) i \theta_{4}\right)^{2}}{\left(e^{16 \sqrt{3} \theta_{4}{ }^{5} i t+\sqrt{3} i \theta_{4} x+\theta_{3}}-\sinh \left(16 t \theta_{4}{ }^{5}-x \theta_{4}-\theta_{6}\right)-\sin \left(16 \theta_{4}{ }^{5} i t-i \theta_{4} x-\theta_{9}\right)\right)^{2}} .
\end{aligned}
$$

3.5. Periodic-Kink Wave Solutions. Here, we will consider the periodic-kink wave solution with selecting the below function which for Equation (1) has been taken as

$$
\begin{gathered}
f=\exp \left(\tau_{1}\right)+\theta_{10} \exp \left(-\tau_{1}\right)+\cosh \left(\tau_{2}\right)+\cos \left(\tau_{3}\right) \\
+\theta_{11}, \tau_{1}=\theta_{1} x+\theta_{2} t+\theta_{3}, \tau_{2}=\theta_{4} x+\theta_{5} t \\
+\theta_{6}, \tau_{3}=\theta_{7} x+\theta_{8} t+\theta_{9}, \\
\Psi(x, t)=\ln (f)_{x x},
\end{gathered}
$$

where $\theta_{i}, i=1, \cdots, 11$, are undetermined amounts which should be detected. Appending (40) into Equation (1) and afterwards collecting the coefficients, we obtain the following consequences:

Case 1.

$$
\theta_{1}=\theta_{4}=i \theta_{7}, \theta_{2}=\theta_{5}=-16 i \theta_{7}^{5}, \theta_{8}=-16 \theta_{7}^{5}, \theta_{11}=0, i^{2}=-1
$$

Substituting (41) into (39) and (40), we achieve a periodic-kink wave solution of Equation (1) which can be written as follows:

$$
\begin{aligned}
& f=e^{-16 i \theta_{7}^{5} t+i \theta_{7} x+\theta_{3}}+\theta_{10} e^{16 i \theta_{7}^{5} t-i \theta_{7} x-\theta_{3}}+\cosh \left(-16 i \theta_{7}^{5} t+i \theta_{7} x+\theta_{6}\right)+\cos \left(-16 \theta_{7}^{5} t+\theta_{7} x+\theta_{9}\right), \\
& \Psi_{2}=\frac{i^{2} \theta_{7}^{2} e^{-16 i \theta_{7}^{5} t+i \theta_{7} x+\theta_{3}}+\theta_{10} i^{2} \theta_{7}^{2} e^{16 i \theta_{7}{ }^{5} t-i \theta_{7} x-\theta_{3}}+\cosh \left(16 i \theta_{7}^{5} t-i \theta_{7} x-\theta_{6}\right) i^{2} \theta_{7}^{2}-\cos \left(16 t \theta_{7}^{5}-x \theta_{7}-\theta_{9}\right) \theta_{7}^{2}}{e^{-16 i \theta_{7}{ }^{5} t+i \theta_{7} x+\theta_{3}}+\theta_{10} e^{16 i \theta_{7}^{5} t-i \theta_{7} x-\theta_{3}}+\cosh \left(16 i \theta_{7}^{5} t-i \theta_{7} x-\theta_{6}\right)+\cos \left(16 t \theta_{7}^{5}-x \theta_{7}-\theta_{9}\right)} \\
& -\frac{\left(i \theta_{7} e^{-16 i \theta_{7}^{5} t+i \theta_{7} x+\theta_{3}}-\theta_{10} i \theta_{7} e^{16 i \theta_{7}^{5} t-i \theta_{7} x-\theta_{3}}-\sinh \left(16 i \theta_{7}^{5} t-i \theta_{7} x-\theta_{6}\right) i \theta_{7}+\sin \left(16 t \theta_{7}^{5}-x \theta_{7}-\theta_{9}\right) \theta_{7}\right)^{2}}{\left(e^{-16 i \theta_{7}^{5} t+i \theta_{7} x+\theta_{3}}+\theta_{10} e^{16 i \theta_{7}^{5} t-i \theta_{7} x-\theta_{3}}+\cosh \left(16 i \theta_{7}^{5} t-i \theta_{7} x-\theta_{6}\right)+\cos \left(16 t \theta_{7}^{5}-x \theta_{7}-\theta_{9}\right)\right)^{2}} .
\end{aligned}
$$


Case 2.

$$
\begin{gathered}
\theta_{1}=\sqrt{3} \theta_{7}=i \sqrt{3} \theta_{4}, \theta_{2}=-\sqrt{3} \theta_{8}=16 \sqrt{3} i \theta_{4}^{5}, \\
\theta_{5}=-16 \theta_{4}^{5}, \theta_{10}=0, \theta_{11}=0, i^{2}=-1 .
\end{gathered}
$$

$$
\begin{aligned}
f= & e^{16 \sqrt{3} \theta_{4}{ }^{5} i t+\sqrt{3} i \theta_{4} x+\theta_{3}}+\sinh \left(-16 t \theta_{4}{ }^{5}+x \theta_{4}+\theta_{6}\right)+\sin \left(-16 \theta_{4}{ }^{5} i t+i \theta_{4} x+\theta_{9}\right), \\
\Psi_{2}= & \frac{3 i^{2} \theta_{4}{ }^{2} e^{16 \sqrt{3} \theta_{4}{ }^{5} i t+\sqrt{3} i \theta_{4} x+\theta_{3}}+\cosh \left(16 t \theta_{4}{ }^{5}-x \theta_{4}-\theta_{6}\right) \theta_{4}{ }^{2}-\cos \left(16 \theta_{4}{ }^{5} i t-i \theta_{4} x-\theta_{9}\right) i^{2} \theta_{4}{ }^{2}}{e^{16 \sqrt{3} \theta_{4}{ }^{5} i t+\sqrt{3} i \theta_{4} x+\theta_{3}}+\cosh \left(16 t \theta_{4}{ }^{5}-x \theta_{4}-\theta_{6}\right)+\cos \left(16 \theta_{4}{ }^{5} i t-i \theta_{4} x-\theta_{9}\right)} \\
& -\frac{\left(\sqrt{3} i \theta_{4} e^{16 \sqrt{3} \theta_{4}{ }^{5} i t+\sqrt{3} i \theta_{4} x+\theta_{3}}-\sinh \left(16 t \theta_{4}{ }^{5}-x \theta_{4}-\theta_{6}\right) \theta_{4}+\sin \left(16 \theta_{4}{ }^{5} i t-i \theta_{4} x-\theta_{9}\right) i \theta_{4}\right)^{2}}{\left(e^{16 \sqrt{3} \theta_{4}{ }^{5} i t+\sqrt{3} i \theta_{4} x+\theta_{3}}+\cosh \left(16 t \theta_{4}{ }^{5}-x \theta_{4}-\theta_{6}\right)+\cos \left(16 \theta_{4}{ }^{5} i t-i \theta_{4} x-\theta_{9}\right)\right)^{2}} .
\end{aligned}
$$

3.6. Periodic Type Wave Solutions-I. Here, we will consider the periodic type wave solution with selecting the below function which for Equation (1) has been taken as

$$
\begin{gathered}
f=\theta_{10} \exp \left(\tau_{1}\right)+\theta_{11} \exp \left(-\tau_{2}\right)+\theta_{12} \cos \left(\tau_{3}\right), \tau_{1}=\theta_{1} x \\
+\theta_{2} t+\theta_{3}, \tau_{2}=\theta_{4} x+\theta_{5} t+\theta_{6}, \tau_{3}=\theta_{7} x+\theta_{8} t+\theta_{9} \\
\Psi(x, t)=\ln (f)_{x x},
\end{gathered}
$$

where $\theta_{i}, i=1, \cdots, 11$, are undetermined amounts which should be detected. Appending (46) into Equation (1) and afterwards collecting the coefficients, we obtain the following consequences:

Case 1.

$$
\theta_{1}=\sqrt{3} \theta_{7}, \theta_{2}=16 \sqrt{3} \theta_{7}^{5}, \theta_{8}=-16 \theta_{7}^{5}, \theta_{11}=0 .
$$

Substituting (47) into (45) and (46), a periodic type wave solution of Equation (1) can be obtained as follows:

$$
\begin{aligned}
f= & \theta_{10} e^{16 \theta_{7}^{5} \sqrt{3} t+\sqrt{3} \theta_{7} x+\theta_{3}}+\theta_{12} \cos \left(16 t \theta_{7}^{5}-x \theta_{7}-\theta_{9}\right), \\
\Psi_{1}= & \frac{3 \theta_{10} \theta_{7}^{2} e^{16 \theta_{7}^{5} \sqrt{3} t+\sqrt{3} \theta_{7} x+\theta_{3}}-\theta_{12} \cos \left(16 t \theta_{7}^{5}-x \theta_{7}-\theta_{9}\right) \theta_{7}^{2}}{\theta_{10} e^{16 \theta_{7}^{5} \sqrt{3} t+\sqrt{3} \theta_{7} x+\theta_{3}}+\theta_{12} \cos \left(16 t \theta_{7}^{5}-x \theta_{7}-\theta_{9}\right)} \\
& -\frac{\left(\theta_{10} \sqrt{3} \theta_{7} e^{16 \theta_{7}^{5} \sqrt{3} t+\sqrt{3} \theta_{7} x+\theta_{3}}+\theta_{12} \sin \left(16 t \theta_{7}^{5}-x \theta_{7}-\theta_{9}\right) \theta_{7}\right)^{2}}{\left(\theta_{10} e^{16 \theta_{7}^{5} \sqrt{3} t+\sqrt{3} \theta_{7} x+\theta_{3}}+\theta_{12} \cos \left(16 t \theta_{7}^{5}-x \theta_{7}-\theta_{9}\right)\right)^{2}} .
\end{aligned}
$$

Case 2.

$$
\theta_{1}=-\theta_{4}=-\sqrt{3} \theta_{7}, \theta_{2}=-\theta_{5}=-16 \sqrt{3} \theta_{7}^{5}, \theta_{8}=-16 \theta_{7}^{5} \text {. }
$$

Substituting (49) into (45) and (46), a periodic type wave solution of Equation (1) can be obtained as follows:

$$
\begin{aligned}
f= & \theta_{10} e^{-16 \theta_{7}^{5} \sqrt{3} t-\sqrt{3} \theta_{7} x+\theta_{3}}+\theta_{11} e^{-16 \theta_{7}^{5} \sqrt{3} t-\sqrt{3} \theta_{7} x-\theta_{6}}+\theta_{12} \cos \left(16 t \theta_{7}^{5}-x \theta_{7}-\theta_{9}\right), \\
\Psi_{2}= & \frac{3 \theta_{10} \theta_{7}^{2} e^{-16 \theta_{7}^{5} \sqrt{3} t-\sqrt{3} \theta_{7} x+\theta_{3}}+3 \theta_{11} \theta_{7}^{2} e^{-16 \theta_{7}^{5} \sqrt{3} t-\sqrt{3} \theta_{7} x-\theta_{6}}-\theta_{12} \cos \left(16 t \theta_{7}^{5}-x \theta_{7}-\theta_{9}\right) \theta_{7}^{2}}{\theta_{10} e^{-16 \theta_{7}^{5} \sqrt{3} t-\sqrt{3} \theta_{7} x+\theta_{3}}+\theta_{11} e^{-16 \theta_{7}^{5} \sqrt{3} t-\sqrt{3} \theta_{7} x-\theta_{6}}+\theta_{12} \cos \left(16 t \theta_{7}^{5}-x \theta_{7}-\theta_{9}\right)} \\
& -\frac{\left(-\theta_{10} \sqrt{3} \theta_{7} e^{-16 \theta_{7}^{5} \sqrt{3} t-\sqrt{3} \theta_{7} x+\theta_{3}}-\theta_{11} \sqrt{3} \theta_{7} e^{-16 \theta_{7}^{5} \sqrt{3} t-\sqrt{3} \theta_{7} x-\theta_{6}}+\theta_{12} \sin \left(16 t \theta_{7}^{5}-x \theta_{7}-\theta_{9}\right) \theta_{7}\right)^{2}}{\left(\theta_{10} e^{-16 \theta_{7}^{5} \sqrt{3} t-\sqrt{3} \theta_{7} x+\theta_{3}}+\theta_{11} e^{-16 \theta_{7}^{5} \sqrt{3} t-\sqrt{3} \theta_{7} x-\theta_{6}}+\theta_{12} \cos \left(16 t \theta_{7}^{5}-x \theta_{7}-\theta_{9}\right)\right)^{2}} .
\end{aligned}
$$

3.7. Periodic Type Wave Solutions-II. Here, we will consider the periodic wave solution with selecting the below function which for Equation (1) has been taken as

$$
\begin{aligned}
f= & \theta_{10} \exp \left(\tau_{1}\right)+\theta_{11} \exp \left(-\tau_{2}\right)+\theta_{12} \sin \left(\tau_{3}\right), \tau_{1}=\theta_{1} x \\
& +\theta_{2} t+\theta_{3}, \tau_{2}=\theta_{4} x+\theta_{5} t+\theta_{6}, \tau_{3}=\theta_{7} x+\theta_{8} t+\theta_{9}
\end{aligned}
$$




$$
\Psi(x, t)=\ln (f)_{x x},
$$

where $\theta_{i}, i=1, \cdots, 11$, are undetermined amounts which should be detected. Appending (52) into Equation (1) and afterwards collecting the coefficients, we obtain the following consequences:

Case 1.

$$
\theta_{1}=\sqrt{3} \theta_{7}, \theta_{2}=16 \sqrt{3} \theta_{7}^{5}, \theta_{8}=-16 \theta_{7}^{5}, \theta_{11}=0 .
$$

Substituting (53) into (51) and (52), a periodic type wave solution of Equation (1) can be obtained as follows:

$$
\begin{aligned}
f= & \theta_{10} e^{16 \theta_{7}^{5} \sqrt{3} t+\sqrt{3} \theta_{7} x+\theta_{3}}+\theta_{12} \sin \left(-16 t \theta_{7}^{5}+x \theta_{7}+\theta_{9}\right), \\
\Psi_{1}= & \frac{3 \theta_{11} \theta_{7}^{2} e^{16 \theta_{7}^{5} \sqrt{3} t+\sqrt{3} \theta_{7} x+\theta_{3}}+\theta_{12} \sin \left(16 t \theta_{7}^{5}-x \theta_{7}-\theta_{9}\right) \theta_{7}^{2}}{\theta_{11} e^{16 \theta_{7}^{5} \sqrt{3} t+\sqrt{3} \theta_{7} x+\theta_{3}}-\theta_{12} \sin \left(16 t \theta_{7}^{5}-x \theta_{7}-\theta_{9}\right)} \\
& -\frac{\left(\theta_{11} \sqrt{3} \theta_{7} e^{16 \theta_{7}^{5} \sqrt{3} t+\sqrt{3} \theta_{7} x+\theta_{3}}+\theta_{12} \cos \left(16 t \theta_{7}^{5}-x \theta_{7}-\theta_{9}\right) \theta_{7}\right)^{2}}{\left(\theta_{11} e^{16 \theta_{7}^{5} \sqrt{3} t+\sqrt{3} \theta_{7} x+\theta_{3}}-\theta_{12} \sin \left(16 t \theta_{7}^{5}-x \theta_{7}-\theta_{9}\right)\right)^{2}} .
\end{aligned}
$$

Case 2.

$$
\theta_{1}=-\theta_{4}=-\sqrt{3} \theta_{7}, \theta_{2}=-\theta_{5}=-16 \sqrt{3} \theta_{7}^{5}, \theta_{8}=-16 \theta_{7}^{5} .
$$

Substituting (55) into (51) and (52), a periodic type wave solution of Equation (1) can be obtained as follows:

$$
\begin{aligned}
& f=\theta_{10} e^{-16 \theta_{7}^{5} \sqrt{3} t-\sqrt{3} \theta_{7} x+\theta_{3}}+\theta_{11} e^{-16 \theta_{7}^{5} \sqrt{3} t-\sqrt{3} \theta_{7} x-\theta_{6}}-\theta_{12} \sin \left(16 t \theta_{7}^{5}-x \theta_{7}-\theta_{9}\right),
\end{aligned}
$$

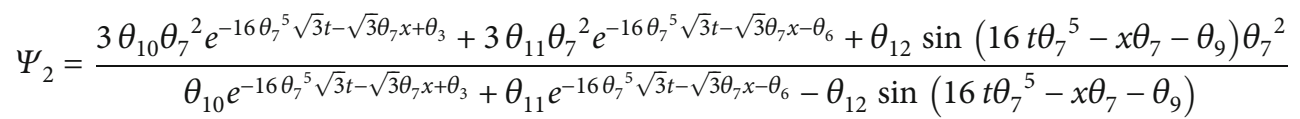

$$
\begin{aligned}
& -\frac{\left(-\theta_{10} \sqrt{3} \theta_{7} e^{-16 \theta_{7}^{5} \sqrt{3} t-\sqrt{3} \theta_{7} x+\theta_{3}}-\theta_{11} \sqrt{3} \theta_{7} e^{-16 \theta_{7}^{5} \sqrt{3} t-\sqrt{3} \theta_{7} x-\theta_{6}}+\theta_{12} \cos \left(16 t \theta_{7}^{5}-x \theta_{7}-\theta_{9}\right) \theta_{7}\right)^{2}}{\left(\theta_{10} e^{-16 \theta_{7}^{5} \sqrt{3} t-\sqrt{3} \theta_{7} x+\theta_{3}}+\theta_{11} e^{-16 \theta_{7}^{5} \sqrt{3} t-\sqrt{3} \theta_{7} x-\theta_{6}}-\theta_{12} \sin \left(16 t \theta_{7}^{5}-x \theta_{7}-\theta_{9}\right)\right)^{2}} .
\end{aligned}
$$

3.8. Solitary Wave Solutions. Here, we will consider the solitary wave solution with selecting the below function which for Equation (1) has been taken as

$$
\begin{gathered}
f=\theta_{7}+\theta_{8} \exp \left(\tau_{1}\right)+\theta_{9} \exp \left(-\tau_{2}\right), \tau_{1}=\theta_{1} x \\
+\theta_{2} t+\theta_{3}, \tau_{2}=\theta_{4} x+\theta_{5} t+\theta_{6}, \\
\Psi(x, t)=\ln (f)_{x x},
\end{gathered}
$$

where $\theta_{i}, i=1, \cdots, 9$, are undetermined amounts which should be detected. Appending (58) into Equation (1) and afterwards collecting the coefficients, we obtain the below consequences:

Case 1.

$$
\begin{aligned}
\theta_{2}= & -\theta_{1}{ }^{5}-5 \theta_{1}{ }^{4} \theta_{4}-10 \theta_{1}{ }^{3} \theta_{4}{ }^{2}-10 \theta_{1}{ }^{2} \theta_{4}{ }^{3} \\
& -5 \theta_{1} \theta_{4}{ }^{4}-\theta_{4}{ }^{5}-\theta_{5}, \theta_{7}=0 .
\end{aligned}
$$

Substituting (59) into (57) and (58), a solitary wave solution of Equation (1) can be written as follows:

$$
\begin{aligned}
f= & \theta_{8} e^{\left(-\theta_{1}^{5}-5 \theta_{1}{ }^{4} \theta_{4}-10 \theta_{1}{ }^{3} \theta_{4}{ }^{2}-10 \theta_{1}{ }^{2} \theta_{4}{ }^{3}-5 \theta_{1} \theta_{4}{ }^{4}-\theta_{4}{ }^{5}-\theta_{5}\right) t+\theta_{1} x+\theta_{3}} \\
& +\theta_{9} e^{-t \theta_{5}-x \theta_{4}-\theta_{6}},
\end{aligned}
$$

$$
\begin{aligned}
\Psi_{1}= & \frac{\theta_{8} \theta_{1}{ }^{2} e^{\left(-\theta_{1}{ }^{5}-5 \theta_{1}{ }^{4} \theta_{4}-10 \theta_{1}{ }^{3} \theta_{4}{ }^{2}-10 \theta_{1}{ }^{2} \theta_{4}{ }^{3}-5 \theta_{1} \theta_{4}{ }^{4}-\theta_{4}{ }^{5}-\theta_{5}\right) t+\theta_{1} x+\theta_{3}}+\theta_{9} \theta_{4}{ }^{2} e^{-t \theta_{5}-x \theta_{4}-\theta_{6}}}{\theta_{8} e^{\left(-\theta_{1}{ }^{5}-5 \theta_{1}{ }^{4} \theta_{4}-10 \theta_{1}{ }^{3} \theta_{4}{ }^{2}-10 \theta_{1}{ }^{2} \theta_{4}{ }^{3}-5 \theta_{1} \theta_{4}{ }^{4}-\theta_{4}{ }^{5}-\theta_{5}\right) t+\theta_{1} x+\theta_{3}}+\theta_{9} e^{-t \theta_{5}-x \theta_{4}-\theta_{6}}} \\
& -\frac{\left(\theta_{8} \theta_{1} e^{\left(-\theta_{1}{ }^{5}-5 \theta_{1}{ }^{4} \theta_{4}-10 \theta_{1}{ }^{3} \theta_{4}{ }^{2}-10 \theta_{1}{ }^{2} \theta_{4}{ }^{3}-5 \theta_{1} \theta_{4}{ }^{4}-\theta_{4}{ }^{5}-\theta_{5}\right) t+\theta_{1} x+\theta_{3}}-\theta_{9} \theta_{4} e^{-t \theta_{5}-x \theta_{4}-\theta_{6}}\right)^{2}}{\left(\theta_{8} e^{\left(-\theta_{1}{ }^{5}-5 \theta_{1}{ }^{4} \theta_{4}-10 \theta_{1}{ }^{3} \theta_{4}{ }^{2}-10 \theta_{1}{ }^{2} \theta_{4}{ }^{3}-5 \theta_{1} \theta_{4}{ }^{4}-\theta_{4}{ }^{5}-\theta_{5}\right) t+\theta_{1} x+\theta_{3}}+\theta_{9} e^{-t \theta_{5}-x \theta_{4}-\theta_{6}}\right)^{2}} .
\end{aligned}
$$

By using suitable values of parameters, the analytical treatment of periodic wave solution is presented in Figure 1 including $3 \mathrm{D}$ plot and $2 \mathrm{D}$ plot with three points of time including $t=0, t=0.02$, and $t=0.04$.

Case 2.

$$
\theta_{1}=-\theta_{4}, \theta_{2}=\theta_{4}^{5}, \theta_{5}=-\theta_{4}^{5} \text {. }
$$

Substituting (62) into (57) and (58), a solitary wave solution of Equation (1) can be obtained as follows:

$$
\begin{aligned}
& f=\theta_{7}+\theta_{8} e^{t \theta_{4}{ }^{5}-x \theta_{4}+\theta_{3}}+\theta_{9} e^{t \theta_{4}{ }^{5}-x \theta_{4}-\theta_{6}}, \\
& \Psi_{2}=\frac{\theta_{4}{ }^{2}\left(\theta_{8} e^{t \theta_{4}{ }^{5}-x \theta_{4}+\theta_{3}}+\theta_{9} e^{t \theta_{4}{ }^{5}-x \theta_{4}-\theta_{6}}\right) \theta_{7}}{\left(\theta_{7}+\theta_{8} e^{t \theta_{4}{ }^{5}-x \theta_{4}+\theta_{3}}+\theta_{9} e^{t \theta_{4}{ }^{5}-x \theta_{4}-\theta_{6}}\right)^{2}} .
\end{aligned}
$$




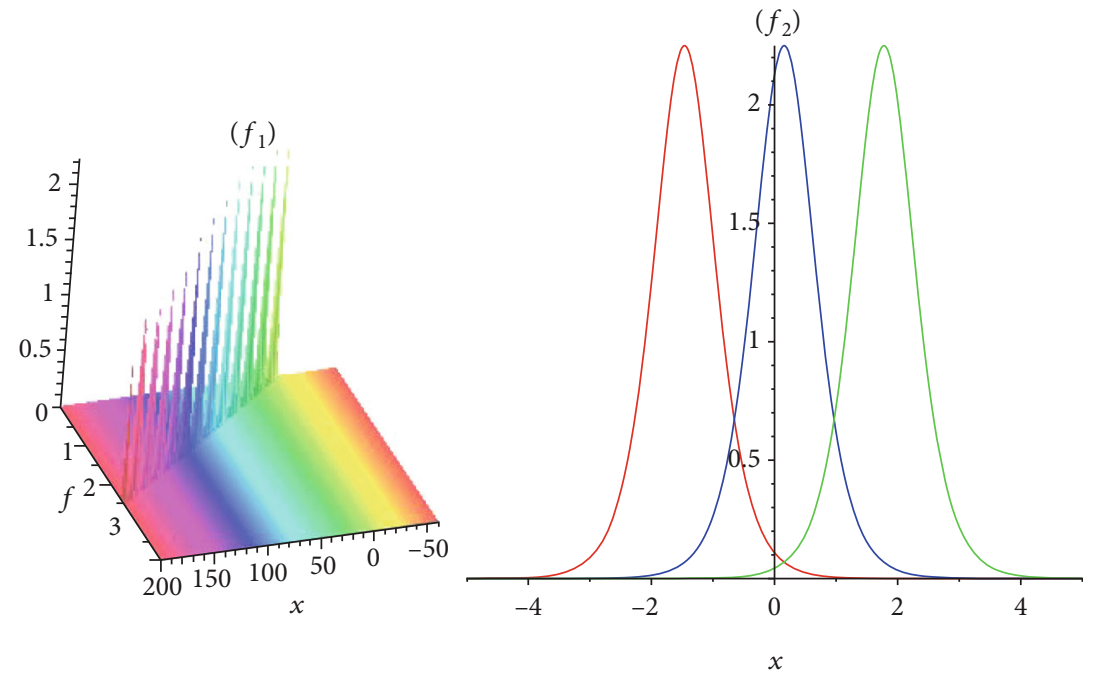

Figure 1: The solitary wave (61) at $\theta_{1}=1, \theta_{4}=2, \theta_{3}=2, \theta_{5}=.5, \theta_{6}=3, \theta_{8}=1.3, \theta_{9}=2.4$.
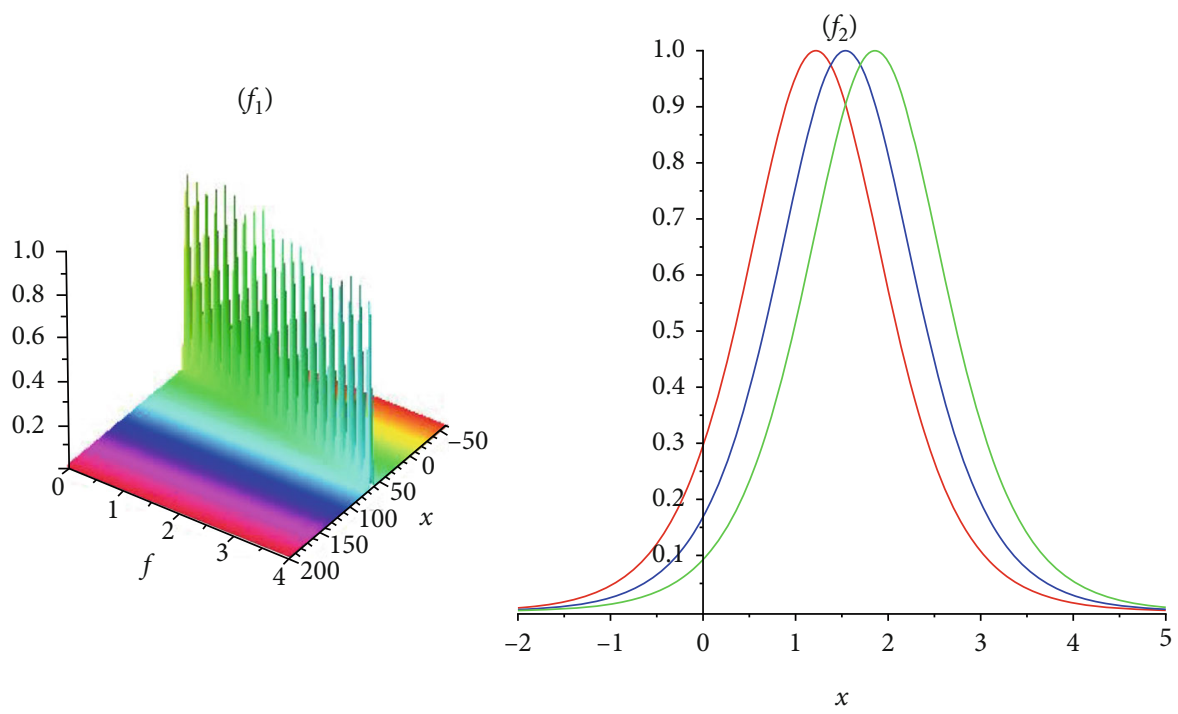

FIGURE 2: The solitary wave $(63)$ at $\theta_{4}=2, \theta_{3}=2, \theta_{6}=3, \theta_{7}=1.3, \theta_{8}=2, \theta_{9}=2.4$.

By using suitable values of parameters, the analytical treatment of periodic wave solution is presented in Figure 2 including 3D plot and $2 \mathrm{D}$ plot with three points of time including $t=0, t=0.02$, and $t=0.04$.

Case 3.

$$
\theta_{1}=-\frac{1}{2}(-1 \pm \sqrt{3} i) \theta_{4}, \theta_{2}=\frac{1}{2}(1 \pm \sqrt{3} i) \theta_{4}^{5}, \theta_{5}=-\theta_{4}^{5}
$$

Substituting (64) into (57) and (58), we achieve a solitary wave solution of Equation (1) as follows:

$$
\begin{aligned}
& f=\theta_{7}+\theta_{8} e^{(1 / 2)) \theta_{4}^{5}(i \sqrt{3}+1)-(1 / 2) x \theta_{4}(i \sqrt{3}-1)+\theta_{3}}+\theta_{9} e^{t \theta_{4}^{5}-x \theta_{4}-\theta_{6}}, \\
& \Psi_{3}=\frac{(1 / 4) \theta_{8} \theta_{4}{ }^{2}(i \sqrt{3}-1)^{2} e^{(1 / 2) t \theta_{4}^{5}(i \sqrt{3}+1)-(1 / 2) x \theta_{4}(i \sqrt{3}-1)+\theta_{3}}+\theta_{9} \theta_{4}{ }^{2} e^{\theta_{4}{ }^{5}-x \theta_{4}-\theta_{6}}}{\theta_{7}+\theta_{8} e^{(1 / 2)) \theta_{4}^{5}(i \sqrt{3}+1)-(1 / 2) \times \theta_{4}(i \sqrt{3}-1)+\theta_{3}}+\theta_{9} \mathrm{e}^{t \theta_{4}^{5}}{ }^{5}-\theta_{4} \theta_{4}-\theta_{6}} \\
& -\frac{\left(-(1 / 2) \theta_{8} \theta_{4}(i \sqrt{3}-1) e^{(1 / 2)) \theta_{4}^{5}(i \sqrt{3}+1)-(1 / 2) x \theta_{4}(i \sqrt{3}-1)+\theta_{3}}-\theta_{9} \theta_{4} e^{t \theta \theta_{4}^{5}-x \theta_{4}-\theta_{6}}\right)^{2}}{\left(\theta_{7}+\theta_{8} e^{(1 / 2) \theta_{4}^{5}(i \sqrt{3}+1)-(1 / 2) x \theta_{4}(i \sqrt{3}-1)+\theta_{3}}+\theta_{9} \mathrm{e}^{\theta_{4}^{5}-x \theta_{4}-\theta_{6}}\right)^{2}} .
\end{aligned}
$$

\section{Stability Analysis of CDG Equation}

According to [59], in order to analyze the propagation characteristics of the rogue wave in detail, we choose the linear stability analysis for the CDG equation via the following 
function along with appropriate parameters:

$$
\Phi(x, t)=\theta+\delta \Omega(x, t)
$$

where the relation constant $\theta$ is a steady state solution of Equation (66). Appending (66) into Equation (1), one can obtain

$$
\begin{aligned}
\delta & \frac{\partial}{\partial t} \Omega(x, t)+\delta \frac{\partial^{5}}{\partial x^{5}} \Omega(x, t)+30 \delta\left(\frac{\partial^{3}}{\partial x^{3}} \Omega(x, t)\right) \theta \\
+ & 30 \delta^{2}\left(\frac{\partial^{3}}{\partial x^{3}} \Omega(x, t)\right) \Omega(x, t)+30 \delta^{2}\left(\frac{\partial}{\partial x} \Omega(x, t)\right) \\
\cdot & \frac{\partial^{2}}{\partial x^{2}} \Omega(x, t)+180 \delta\left(\frac{\partial}{\partial x} \Omega(x, t)\right) \theta^{2}+360 \delta^{2}\left(\frac{\partial}{\partial x} \Omega(x, t)\right) \\
\cdot & \theta \Omega(x, t)+180 \delta^{3}\left(\frac{\partial}{\partial x} \Omega(x, t)\right)(\Omega(x, t))^{2}=0 .
\end{aligned}
$$

By linearization of Equation (67), we get

$$
\begin{aligned}
& \delta \frac{\partial}{\partial t} \Omega(x, t)+\delta \frac{\partial^{5}}{\partial x^{5}} \Omega(x, t)+30 \delta\left(\frac{\partial^{3}}{\partial x^{3}} \Omega(x, t)\right) \theta \\
& \quad+180 \delta\left(\frac{\partial}{\partial x} \Omega(x, t)\right) \theta^{2}=0 .
\end{aligned}
$$

Theorem 1. Presume that the solution of Equation (68) has the following form:

$$
\Omega(x, t)=\rho_{1} e^{i(\alpha x+\beta t)},
$$

where $\alpha, \beta$ are the normalized wave numbers, by putting (69) into Equation (68), then by solving for $\beta$, we can achieve the following form

$$
\beta(\alpha)=-\alpha^{5}+30 \alpha^{3} \theta-180 \alpha \theta^{2} .
$$

Proof. By appending the equality (69) in the linear PDE (68), we obtain

$$
\begin{aligned}
& \delta \frac{\partial}{\partial t} \Omega(x, t)+\delta \frac{\partial^{5}}{\partial x^{5}} \Omega(x, t)+30 \delta\left(\frac{\partial^{3}}{\partial x^{3}} \Omega(x, t)\right) \theta \\
& +180 \delta\left(\frac{\partial}{\partial x} \Omega(x, t)\right) \theta^{2}=i \mathrm{e}^{i(\alpha x+\beta t)} \\
& \cdot \delta \rho_{1}\left(\alpha^{5}-30 \alpha^{3} \theta+180 \alpha \theta^{2}+\beta\right)=0 .
\end{aligned}
$$

By solving and simplifying, we can find the value of $\beta(\alpha)$ as follows:

$$
\beta(\alpha)=-\alpha^{5}+30 \alpha^{3} \theta-180 \alpha \theta^{2} .
$$

After that, we get to the needed solution. Hence, the proof of the theorem is complete.

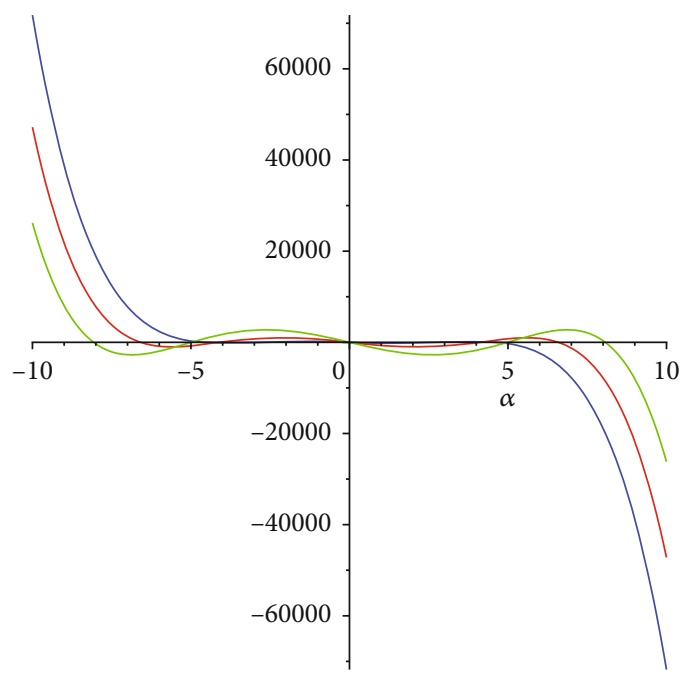

FIGURE 3: The behavior of stability analysis $\beta(\alpha)$ and wave number $\alpha$ with the disparate amounts $\theta=1, \theta=2, \theta=3$.

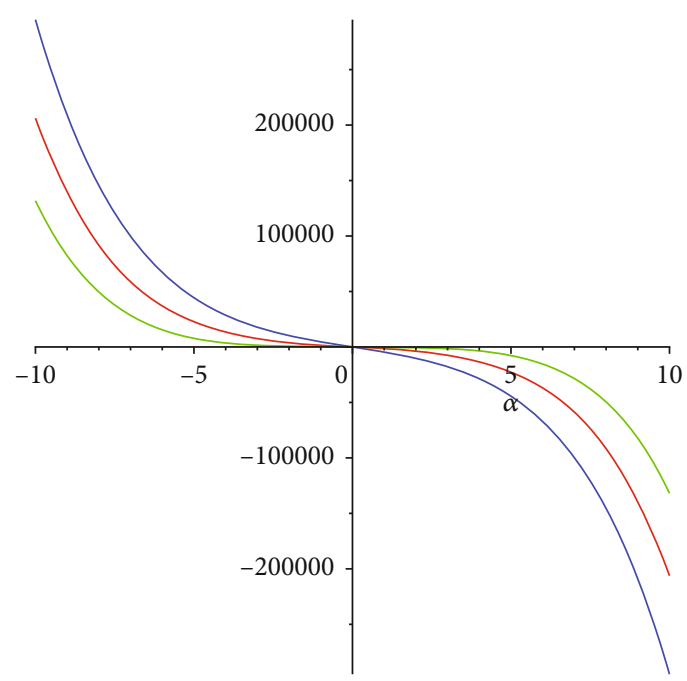

FIgURE 4: The behavior of stability analysis $\beta(\alpha)$ and wave number $\alpha$ with the disparate amounts $\theta=-5, \theta=-3, \theta=-1$.

In Figures 3-5, it can be seen that when the sign of $\beta(\alpha)$ is positive for all amounts of $\alpha$, then any superposition of solutions of the form $e^{i(\alpha x+\beta t)}$ will come to ascent, while the sign of $\beta(\alpha)$ is negative for all amounts of $\alpha$, then any superposition of solutions of the form $e^{i(\alpha x+\beta t)}$ will come to decay and the steady condition is stable. After that, in Figures 3 and 4, it can be observed that if the $\beta(\alpha)$ is positive or negative for some amounts of $\alpha$, then with increasing time some components of a superposition will become descent, and the steady condition is stable. Finally, in Figure 5, it can be perceived that when the sign of $\beta(\alpha)$ is positive for all amounts of $\alpha$, then any superposition of solutions of the form $e^{i(\alpha x+\beta t)}$ will come to ascent, while the sign of $\beta(\alpha)$ is negative for all amounts of $\alpha$, then any superposition of solutions of the form $e^{\mathrm{i}(\alpha x+\beta t)}$ will come to decay and the steady condition is stable. 


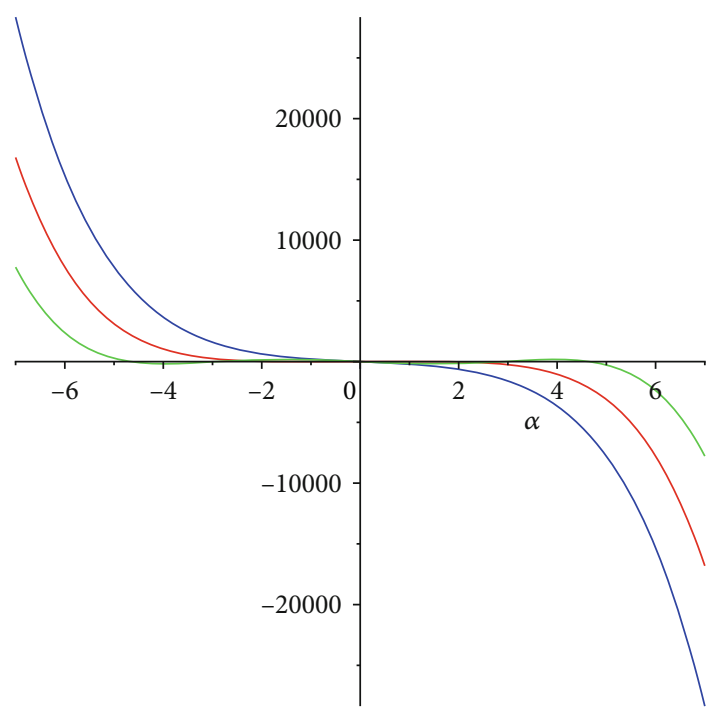

FIgURE 5: The behavior of stability analysis $\beta(\alpha)$ and wave number $\alpha$ with the disparate amounts $\theta=-1, \theta=0, \theta=1$.

\section{Conclusion}

In this work, the multiple exp-function, cross-kink, periodickink, and solitary wave methods with predictability of the $(1+1)$-dimensional CDG equation are investigated with more arbitrary autocephalous parameters. It is not hard to see that the general periodic-kink solution is an algebraically wave solution, and we noticed that some obtained solutions are singular periodic solitary wave solution which is periodic wave or periodic-kink, or solitary wave solutions in $x-t$ direction. Also, the other presented solution is a breather type of two-solitary wave solution which contains a periodic wave and two solitary waves, whose amplitude periodically oscillates with the evolution of time. Moreover, the kink and periodic solutions were analyzed and investigated. In addition, the periodic-kink waves appeared when the periodic solution cut by a stripe soliton before or after a special time. Meanwhile, the modulation instability was applied to discuss the stability of earned solutions. Finally, we show some graphs to explain these solutions.

\section{Data Availability}

The datasets supporting the conclusions of this article are included within the article and its additional file.

\section{Conflicts of Interest}

The authors declare that they have no conflicts of interest.

\section{Acknowledgments}

This project was supported by Researchers Supporting Project number (RSP-2020/210), King Saud University, Riyadh, Saudi Arabia.

\section{References}

[1] M. S. Osman, H. Rezazadeh, and M. Eslami, "Traveling wave solutions for $(3+1)$ dimensional conformable fractional Zakharov-Kuznetsov equation with power law nonlinearity," Nonlinear Engineering, vol. 8, no. 1, pp. 559-567, 2019.

[2] D. Lu, K. U. Tariq, M. S. Osman, D. Baleanu, M. Younis, and M. M. A. Khater, "New analytical wave structures for the (3 +1)-dimensional Kadomtsev- Petviashvili and the generalized Boussinesq models and their applications," Results in Physics, vol. 14, p. 102491, 2019.

[3] D. Kumar, C. Park, N. Tamanna, G. C. Paul, and M. S. Osman, "Dynamics of two-mode Sawada-Kotera equation: mathematical and graphical analysis of its dual-wave solutions," Results in Physics, vol. 19, article 103581, 2020.

[4] Y. Ding, M. S. Osman, and A. M. Wazwaz, "Abundant complex wave solutions for the nonautonomous Fokas-Lenells equation in presence of perturbation terms," Optik, vol. 181, pp. 503-513, 2019.

[5] M. S. Osman, M. Inc, J. G. Liu, K. Hosseini, and A. Yusuf, "Different wave structures and stability analysis for the generalized (2+1)-dimensional Camassa-Holm-Kadomtsev-Petviashvili equation," Physica Scripta, vol. 95, no. 3, article 035229, 2020.

[6] V. S. Kumar, H. Rezazadeh, M. Eslami, F. Izadi, and M. S. Osman, "Jacobi elliptic function expansion method for solving $\mathrm{KdV}$ equation with conformable derivative and dual-power law nonlinearity," International Journal of Applied and Computational, vol. 5, no. 5, p. 127, 2019.

[7] F. Yu and L. Li, "Inverse scattering transformation and soliton stability for a nonlinear Gross- Pitaevskii equation with external potentials," Applied Mathematics Letters, vol. 91, pp. 4147, 2019.

[8] A. Ramani, "Inverse scattering, ordinary differential equations of painlevé-type, and Hirota's bilinear formalism," Annals of the New York Academy of Sciences, vol. 373, no. 1, pp. 54-67, 1981.

[9] Y. Li, R. Li, B. Xue, and X. Geng, "A generalized complex $\mathrm{mKdV}$ equation: Darboux transformations and explicit solutions," Wave Motion, vol. 98, article 102639, 2020.

[10] B. Q. Li and Y. L. Ma, "Extended generalized Darboux transformation to hybrid rogue wave and breather solutions for a nonlinear Schrodinger equation," Applied Mathematics and Computation, vol. 386, article 125469, 2020.

[11] X. W. Yan, "Lax pair, Darboux-dressing transformation and localized waves of the coupled mixed derivative nonlinear Schrodinger equation in a birefringent optical fiber," Applied Mathematics Letters, vol. 107, article 106414, 2020.

[12] M. S. Osman, D. Baleanu, A. R. Adem, K. Hosseini, M. Mirzazadeh, and M. Eslami, "Double-wave solutions and Lie symmetry analysis to the $(2+1)$-dimensional coupled Burgers equations," Chinese Journal of Physics, vol. 63, pp. 122129, 2020.

[13] R. Hirota, The Direct Method in Soliton Theory, Cambridge University Press, 2009.

[14] M. S. Osman and H. I. Abdel-Gawad, "Multi-wave solutions of the (2+1)-dimensional Nizhnik-Novikov-Veselov equations with variable coefficients," The European Physical Journal Plus, vol. 130, no. 10, p. 215, 2015.

[15] J. G. Liu, W. H. Zhu, M. S. Osman, and W. X. Ma, “An explicit plethora of different classes of interactive lump solutions for an extension form of 3D-Jimbo-Miwa model," The European Physical Journal Plus, vol. 135, no. 5, p. 412, 2015. 
[16] W. X. Ma, Y. Zhou, and R. Dougherty, "Lump-type solutions to nonlinear differential equations derived from generalized bilinear equations," International Journal of Modern Physics $B$, vol. 30, no. 28 n29, article 1640018, 2016.

[17] J. G. Liu, M. S. Osman, W. H. Zhu, L. Zhou, and D. Baleanu, "The general bilinear techniques for studying the propagation of mixed-type periodic and lump-type solutions in a homogenous-dispersive medium," AIP Advances, vol. 10, no. 10, article 105325, 2020.

[18] C. J. Wang, "Spatiotemporal deformation of lump solution to (2+1)-dimensional KdV equation," Nonlinear Dynamics, vol. 84, no. 2, pp. 697-702, 2016.

[19] M. R. Foroutan, J. Manafian, and A. Ranjbaran, "Lump solution and its interaction to $(3+1)-\mathrm{D}$ potential-YTSF equation," Nonlinear Dynamics, vol. 92, no. 4, pp. 20772092, 2018.

[20] L. Kaur and A. M. Wazwaz, "Lump, breather and solitary wave solutions to new reduced form of the generalized BKP equation," International Journal of Numerical Methods for Heat and Fluid Flow, vol. 29, no. 2, pp. 569-579, 2019.

[21] L. Kaur and A. M. Wazwaz, "Bright-dark lump wave solutions for a new form of the (3+1)-dimensional BKP-Boussinesq equation," Romanian Reports in Physics, vol. 71, pp. 1-11, 2019.

[22] L. Kaur and A. M. Wazwaz, "Dynamical analysis of lump solutions for $(3+1)$ dimensional generalized KP-Boussinesq equation and its dimensionally reduced equations," Physica Scripta, vol. 93, no. 7, article 075203, 2018.

[23] S. Singh, L. Kaur, K. Sakkaravarthi, R. Sakthivel, and K. Murugesan, "Dynamics of higher-order bright and dark rogue waves in a new $(2+1)$-dimensional integrable Boussinesq model," Physica Scripta, vol. 95, no. 11, article 115213, 2020.

[24] J. Manafian, B. Mohammadi Ivatlo, and M. Abapour, "Lumptype solutions and interaction phenomenon to the $(2+1)$ dimensional breaking soliton equation," Applied Mathematics and Computation, vol. 13, pp. 13-41, 2019.

[25] O. A. Ilhan and J. Manafian, "Periodic type and periodic crosskink wave solutions to the $(2+1)$-dimensional breaking soliton equation arising in fluid dynamics," Modern Physics Letters B, vol. 33, no. 23, article 1950277, 2019.

[26] L. L. Huang and Y. Chen, "Lump solutions and interaction phenomenon for $(2+1)$-dimensional Sawada-Kotera equation," Communications in Theoretical Physics, vol. 67, no. 5, pp. 473-478, 2017.

[27] J. Q. Lu and S. D. Bilige, "Lump solutions of a (2+1)-dimensional bSK equation," Nonlinear Dynamics, vol. 90, no. 3, pp. 2119-2124, 2017.

[28] J. Manafian and M. Lakestani, "Lump-type solutions and interaction phenomenon to the bidirectional Sawada-Kotera equation," Pramana, vol. 92, no. 3, p. 41, 2019.

[29] J. Zhao, J. Manafian, N. E. Zaya, and S. A. Mohammed, "Multiple rogue wave, lump-periodic, lump-soliton, and interaction betweenk-lump andk-stripe soliton solutions for the generalized KP equation," Mathematical Methods in the Applied Sciences, 2020.

[30] J. Manafian, S. A. Mohammed, A. Alizadeh, H. M. Baskonus, and W. Gao, "Investigating lump and its interaction for the third-order evolution equation arising propagation of long waves over shallow water," European Journal of Mechanics B/Fluids, vol. 84, pp. 289-301, 2020.
[31] B. He and Q. Meng, "Bilinear form and new interaction solutions for the sixth-order Ramani equation," Applied Mathematics Letters, vol. 98, pp. 411-418, 2019.

[32] J. Manafian and M. Lakestani, "N-lump and interaction solutions of localized waves to the $(2+1)$-dimensional variablecoefficient Caudrey-Dodd-Gibbon-Kotera-Sawada equation," Journal of Geometry and Physics, vol. 150, p. 103598, 2020.

[33] J. Wang, H. L. An, and B. Li, "Non-traveling lump solutions and mixed lump kink solutions to $(2+1)$-dimensional variable-coefficient Caudrey Dodd Gibbon Kotera Sawada equation," Modern Physics Letters B, vol. 33, no. 22, article 1950262, 2019.

[34] Z. D. Dai, J. Liu, X. P. Zeng, and Z. J. Liu, "Periodic kink-wave and kinky periodic-wave solutions for the Jimbo-Miwa equation," Physics Letters A, vol. 372, no. 38, pp. 5984-5986, 2008.

[35] W. X. Ma and Z. Zhu, "Solving the $(3+1)$-dimensional generalized KP and BKP equations by the multiple exp-function algorithm," Applied Mathematics and Computation, vol. 218, no. 24, pp. 11871-11879, 2012.

[36] R. N. Aiyer, B. Fuchssteiner, and W. Oevel, "Solitons and discrete eigenfunctions of the recursion operator of non-linear evolution equations. I. the Caudrey-Dodd-Gibbon-SawadaKotera equation," Journal of Physics A: Mathematical and General, vol. 19, no. 18, pp. 3755-3770, 1986.

[37] Y. G. Xu, X. W. Zhou, and L. Yao, "Solving the fifth order Caudrey-Dodd-Gibbon (CDG) equation using the exp- function method," Applied Mathematics and Computation, vol. 206, no. 1, pp. 70-73, 2008.

[38] A. M. Wazwaz, "Analytic study of the fifth order integrable nonlinear evolution equations by using the tanh method," Applied Mathematics and Computation, vol. 174, no. 1, pp. 289-299, 2006.

[39] A. M. Wazwaz, "Multiple-soliton solutions for the fifth order Caudrey-Dodd-Gibbon (CDG) equation," Applied Mathematics and Computation, vol. 197, no. 2, pp. 719-724, 2008.

[40] C. Cao, Y. Wu, and X. Geng, "On quasi-periodic solutions of the $2+1$ dimensional Caudrey-Dodd-Gibbon-KoteraSawada equation," Physics Letters A, vol. 256, no. 1, pp. 59-65, 1999.

[41] S. Y. Lou, "Twelve sets of symmetries of the Caudrey-DoddGibbon-Sawada-Kotera equation," Physics Letters A, vol. 175, no. 1, pp. 23-26, 1993.

[42] J. H. He, "New interpretation of homotopy perturbation method," International Journal of Modern Physics B, vol. 20, pp. 2561-2568, 2012.

[43] H. Tari, D. D. Ganji, and M. Rostamian, "Approximate solutions of $\mathrm{K}(2,2), \mathrm{KdV}$ and modified $\mathrm{KdV}$ equations by variational iteration method, homotopy perturbation method and homotopy analysis method," International Journal of Nonlinear Sciences and Numerical Simulation, vol. 8, pp. 203-210, 2007.

[44] T. Ozis and A. Yildirim, "Traveling wave solution of Korteweg-de Vries equation using He's homotopy perturbation method," International Journal of Nonlinear Sciences and Numerical Simulation, vol. 8, pp. 239-242, 2007.

[45] A. H. Salas, O. G. Hurtado, and J. E. C. Hernández, “Computing multi-soliton solutions to Caudrey-Dodd- Gibbon equation by Hirota's method," Eur. Int. J. Phys. Sc., vol. 6, no. 34, 2011.

[46] A. Yusuf, T. A. Sulaiman, M. Inc, and M. Bayram, "Breather wave, lump-periodic solutions and some other interaction 
phenomena to the Caudrey-Dodd-Gibbon equation," The European Physical Journal Plus, vol. 135, no. 7, p. 563, 2020.

[47] D. Kumar and S. Kumar, "Some more solutions of CaudreyDodd-Gibbon equation using optimal system of lie symmetries," International Journal of Applied and Computational Mathematics, vol. 6, no. 4, p. 125, 2020.

[48] D. Kumar and S. Kumar, "Solitary wave solutions of pZK equation using Lie point symmetries," The European Physical Journal Plus, vol. 135, p. 162, 2020.

[49] S. Kumar, W. X. Ma, and A. Kumar, "Lie symmetries, optimal system and group-invariant solutions of the $(3+1)$-dimensional generalized KP equation," Chinese Journal of Physics, vol. 69, pp. 1-23, 2021.

[50] S. Kumar, D. Kumar, and A. Kumar, "Lie symmetry analysis for obtaining the abundant exact solutions, optimal system and dynamics of solitons for a higher-dimensional Fokas equation," Chaos, Solitons \& Fractals, vol. 29, p. 110507, 2020.

[51] S. Kumar, M. Niwas, and A. M. Wazwaz, "Lie symmetry analysis, exact analytical solutions and dynamics of solitons for (2 +1)-dimensional NNV equations," Physica Scripta, vol. 95, no. 9, article 095204, 2020.

[52] S. Kumar and D. Kumar, "Lie symmetry analysis, complex and singular solutions of (2+1)-dimensional combined MCBSnMCBS equation," International Journal of Dynamics and Control, vol. 7, no. 2, pp. 496-509, 2019.

[53] S. Kumar and S. Rani, "Lie symmetry reductions and dynamics of soliton solutions of (2+1)-dimensional Pavlov equation," Pramana, vol. 94, no. 1, p. 116, 2020.

[54] L. Kaur and A. M. Wazwaz, "Bright-dark optical solitons for Schrödinger-Hirota equation with variable coefficients," Optik, vol. 179, pp. 479-484, 2019.

[55] W. Gao, H. F. Ismael, H. Bulut, and H. M. Baskonus, "Instability modulation for the (2+1)-dimension paraxial wave equation and its new optical soliton solutions in Kerr media," Physica Scripta, vol. 95, no. 3, article 035207, 2020.

[56] W. Gao, P. Veeresha, D. G. Prakasha, H. M. Baskonus, and G. Yel, "A powerful approach for fractional DrinfeldSokolov-Wilson equation with Mittag-Leffler law," Alexandria Engineering Journal, vol. 58, no. 4, pp. 1301-1311, 2019.

[57] J. Manafian, "Novel solitary wave solutions for the $(3+1)$ dimensional extended Jimbo-Miwa equations," Computers \& Mathematcs with Applications, vol. 76, no. 5, pp. 1246-1260, 2018.

[58] O. İlhan, S. G. Kasimov, S. Q. Otaev, and H. M. Baskonus, "On the solvability of a mixed problem for a high-order partial differential equation with fractional derivatives with respect to time, with Laplace operators with spatial variables and nonlocal boundary conditions in Sobolev classes," Mathematics, vol. 7, no. 3, p. 235, 2019.

[59] J. Manafian, O. A. Ilhan, and A. Alizadeh, "Periodic wave solutions and stability analysis for the KP-BBM equation with abundant novel interaction solutions," Physica Scripta, vol. 95 , no. 6 , article $065203,2020$.

[60] J. Manafian, B. Mohammadi Ivatlo, and M. Abapour, "Breather wave, periodic, and cross-kink solutions to the generalized Bogoyavlensky-Konopelchenko equation," Mathematical Methods in the Applied Sciences, vol. 43, no. 4, pp. 1753-1774, 2019.

[61] Y. Yakup and Y. Emrullah, "Multiple exp-function method for soliton solutions of nonlinear evolution equations," Chinese Physics B, vol. 26, no. 7, pp. 20-26, 2017.
[62] A. G. Alnowehy, "The multiple exp-function method and the linear superposition principle for solving the $(2+1)$-dimensional Calogero-Bogoyavlenskii-Schiff equation," Zeitschrift für Naturforschung A, vol. 70, no. 9, pp. 775-779, 2015.

[63] R. A. Abdullahi, "The generalized (1+1)-dimensional and (2 +1 )-dimensional Ito equations: multiple exp-function algorithm and multiple wave solutions," Computers \& Mathematcs with Applications, vol. 71, pp. 1248-1258, 2016. 